\title{
NSIS Extensions: An End-to-End QoS Aware Signaling Protocol for NGN
}

\author{
Tahir Mehmood and Sathiamoorthy Manoharan
}

\begin{abstract}
The Next generation network (NGN) deployment is in its infancy. With great technological advancement in internetworking infrastructure, NGN faces many challenges in the form of heterogeneity in networking and user expectations such as higher bandwidth, seamless mobility, internetworking protocols, multicast addressing (or multicasting), security and a guaranteed Quality of Service $(\mathrm{QoS})$. This paper investigates the major challenges being faced during the transition of current internetwork into the future generation network i.e. NGN. We will look into IETF's efforts on developing signaling protocols in satisfying the ever demanding end-toend guaranteed QoS needs by end users or interconnected network nodes. A comparative study is done for Integrated Services (IntServ), Differentiated Services (DiffServ), Resource Reservation Protocol (RSVP), and the newly developed Next Steps in Signaling (NSIS) as QoS signaling protocol and NSIS possible extensions.
\end{abstract}

Index Terms-QoS, NGN, RSVP, NSIS, QoS NSLP.

\section{INTRODUCTION}

The use of signaling in computer network has helped to implement controls related to routing, billing, QoS and efficient use of bandwidth. SS7 signaling by ITU-T has been used by PSTN based telephony industry since 1977. After the launch of Internet, IP based protocols and signaling control was introduced. IETF's efforts included creation of IntServ, DiffServ and the current internet standard IP QoS signaling protocol i.e. RSVP. Since all these signaling protocols were meant to resolve current or some future needs, hence they did not have a scalable design to accommodate the QoS signaling needs of the Next Generation Networks (NGN). The per-session control by IntServ and DiffServ's best-effort QoS enabled architecture for efficient low-latency services are some remarkable achievements by IETF.

With the evolution of NGN, some new issues evolved related to provisioning of QoS guaranteed transmission of voice and multimedia over IP. Integrated Services (IntServ) and Differentiated Services (DiffServ) failed to provide end2-end QoS service because they were designed without keeping mobility support in focus [1]. Some extensions in DiffServ based models also tried to cope with the demands of modern IP multimedia and voice traffic, but they could not live up to desired industry standards for future networks needs. IETF has started working on NSIS Next Steps in Signaling (NSIS) framework in 2005 [2]. The main aim of

Manuscript received August 5, 2012; revised October 24, 2012.

The authors are with the Department of Computer Science, Tamaki Campus, University of Auckland, New Zealand (e-mail: terryraja@yahoo.com). such signaling was to provide and control information about data flow along its path during network transmission. A study on requirement of such architecture was presented in [3]. The key goals of NSIS could be summarised as: information availability - when and where needed; creating a modular design to allow future extensions; decoupling of protocol and information layer - which means work with routers that support NSIS and ignore the rest.

This paper will mainly look into the achievements of IETF in developing end-2-end QoS aware signaling architecture and its further extensions like QoS NSLP, QSPEC Template etc. A review on signaling and QoS signaling need will be presented, looking at the work done for standardisation of such protocols by IETF and ITU-T. We will also compare the key differences of IntServ, DiffServ, RSVP, NSIS and its extensions' architecture.

The rest of the paper is organised as follows: Section-II will discuss the background studies on signaling protocols for communications. Section-III will present literature survey on IETF' and ITU-T standardization efforts on NGN architecture and signaling protocols. In Section-IV we will compare the architectural differences and services offered by the protocols developed so far for IP based internet telephony (e.g. VoIP) and multimedia. In Section-V we will present a critical analysis of possible NSIS extensions. Finally, we will give conclusion of our paper in Section-VI.

\section{BACKGROUND STUDY}

The current internet structure has been developed into a complex heterogeneous kind of global information sharing architecture. The deployment of WiMAX, LANs/WLANs, WANs, MANs and Ad Hoc networks has brought a revolution in communication industry. The demand for mobility and bandwidth with QoS guaranteed delivery of IP based multimedia and telephony have posed greatest challenges to the research community on provisioning of protocols and standards that would fulfill the demands for end-to-end QoS. The creation of IMS, 3GPP, NGN research groups at IETF and ITU-T, are some of the research efforts that are working on designing and standardization of such protocols.

Next generation networks (NGNs), are packet-based networks that provide QoS enabled transport technologies both for telecommunication services and broadband network services. In order to provide QoS for communication services, NGN works independent of underlying transport technologies. NGN supports mobility and convergence between fixed and mobile networks [4]. Another definition for NGN could be QoS enabled IP-based networking framework, used for transportation of data, voice and video 
[5]. Some authors referred NGN as migration from separately existing independent networks towards a converged IP based network, extending support to different sets of traffic such as voice, video and data [6].

At the core of NGN, IMS framework is used for managing and controlling multimedia sessions over IP networks and could be thought of as a part of service delivery platform for IP-base telecommunication services [7]. The main features of IMS are considered to be handle multimedia sessions; intelligent services initialization at application server, provision of network related data to IMS based application and helping applications in using network functionalities via open standardized interfaces. Three main functional areas of MIS are user, control and application. IMS-TISPAN is a model designed to help practical implementation of NGN in telecom networks, giving telecom operators an option to use this architecture for their convergence into NGN. More on NGN and its working with IMS-TISPAN could be found in [8].

The introduction of voice and multimedia services in NGN has also increased the demand for a QoS based architecture of NGN services. QoS of a network means it should provide better services to network traffic without depending on the underlying protocols or technologies [9]. The design and standardization of protocols such as IntServ, DiffServ and multiprotocol label switching (MPLS), took place to provide the QoS demands for real time applications' data. IntServ provides per flow guarantees but lacks scalability. DiffServ, is more scalable, but provides only service differentiation for large network traffic types.

MPLS provides per flow guarantees but it's a complex scheme as compared to the rest. For instance, the MPLS domain routers have to run different type of routing algorithms to look for the best QoS paths available. Hence the need of a more generic QoS protocol that could meet the requirements of NGN' converged environment eventually evolved. NSIS and its extensions are the current trends that are promising some resolution to the problems of end-to-end QoS for telecom networks. But still, a lot more effort is required to have a fully operational and functional QoS protocol, fulfilling all or most of the future generation networks requirements. The next section will present a literature survey on IETF' and ITU-T standardization efforts on signaling protocols and NGN QoS architecture.

\section{LITERATURE SURVEY}

Due to NGN' interworking with external IP-based networks, and mobility of user nodes; end-to-end QoS provision has become a critical issue. The literature survey will focus IETF's efforts in developing a reliable and scalable protocol for end-2-end QoS in future networks.

According to the authors of [9], IETF's Integrated service (IntServ) framework aimed at providing per-flow QoS guarantees to single application session. IntServ requires end applications to request the QoS they require from routers, while RSVP (resource reservation protocol) is used to establish and maintain a sender-receiver connection that guarantees some level of service. The issue with IntServ/RSVP architecture is that it's not scalable, because of more storage needs and processing demands and routers involvement to implement RSVP, admission control, classification and packet scheduling. Differentiated Services (DiffServ) on other hand, is more scalable, manageable and easily deployable for service differentiation in IP networks. The complexity is shifted to the routers on the ends and the core routers would be kept free for routing tasks. DiffServ is better than IntServ in scalability and configurability; it has the issues of not being able to maintain multiple paths, hence not a strong candidate in the better QoS provision in converged networks.

Jarmo Harju and Perttu [10] presented a comparison of IntServ and DiffServ. Their studies results proved that DiffServ QoS measures were better than IntServ. The main concern of DiffServ is scalability and its maintenance overhead in heavy multimedia traffic networks. Some scalability is attained through aggregation of the traffic classification state, which actually improved the QoS characteristics due to the smaller processing overhead. E-H Cho et.al also preferred DiffServ for the development of IP QoS in core networks because of the built-in scalability problems of IntServ [11].

Z. Mammeri [12] recommended that in order to deliver QoS guarantees in the future Internet, combining the capabilities of IntServ and DiffServ architectures might resolve some of key issues. He looked into mapping between user QoS requirements, Service Level Agreements (SLAs) and DiffServ codepoints and presented a general framework for the analysis of QoS mapping on the basis of per domain and per region behavior. The use of IntServ over DiffServ networks is more complex for multicast as compared to the unicast sessions. Selection and coordination of multiple paths with many QoS requirements according to characteristics of the receivers of the same multicast flow is of great importance. This in turn will affect the SLAs as well. Problems such as, handling multicast flows, methodology to steer QoS mapping criteria and the validation in live scenarios were not discussed by the authors.

QoS support in broadband access network is very important due to real-time multimedia applications such as voice over IP, teleconferencing, IP TV, and audio/video streaming. All these applications are always in demand of strictest QoS. Most of these services are currently being deployed in broadband access networks due to large spread and already existence of broadband infrastructure.

S. Park [13] proposed a few enhancements for DiffServ QoS in broadband access network environment. His static models are; Flat DiffServ working for per-subscriber QoS and Structured DiffServ giving per-service and persubscriber DiffServ QoS. For dynamic DiffServ models, the Park proposed Direct DiffServ for peer-to-peer multimedia application and Indirect DiffServ for ISPs (Internet Service Provider) applications. But the paper fails to address the integration of this architecture in NGN and converged environment.

The drawback of using IntServ and DiffServ for a particular type of network traffic or environment rather than more generic internet communication professed the need for more generic QoS architecture for future generation networks. That's what organizations like IETF and ITU-T started working on more general protocols for 
heterogeneous future generation networks, keeping mobility in focus.

María Ángeles, et.al. in their work [14] have studied the problems faced during the design, implementation and validation of the EuQoS system and recommended some main steps to be taken for smooth transition to NGN. The following is the summary of their analysis:

- End users' requirements must be given due importance in writing NGN specifications.

- The integration of service platforms as the only way to provide QoS is a complex solution and more scalable solution should be devised.

- The specification should be on time before commercial systems are implemented and deployed. This eventually will save the efforts that will be wasted otherwise after largescale deployment of future heterogeneous networks.

Signaling protocols could either be working using a hard state or a soft state. Hard state is set up in clients upon receipt of a setup-message and stripped-off only upon receipt of an explicit tear-down message [1]. Examples of hard state protocols are SS7 and ST-2; SS7 being telecom industry standard for many years. In soft state protocols, nonpermanent control state is used in network clients that wouldn't be saved unless refreshing occurs. Soft state was first formalized in RSVP. Signaling built on the soft state has been used by many other researchers for their proposed schemes [15].

Intserv, Diffserv and RSVP could have been glued together and formed a standard solution for QoS, but this never happened due to their built-in issues of handling diversity of future generation networks. S Wei et.al.[16] highlighted an important challenge for router vendors, that is to design such routers which are RSVP-capable, rendering support to mixed services models in a flexible manner. They proposed a model of an experimental RSVP-capable router that would provide transportation of integrated traffic of different kind of services. Their analysis shows that inclusion of an intelligent RSVP daemon and management agent in routers would enable to provide flexible and reliable support for multiple QoS services. But this model demands an upgrade in routers physical structure, which would never be feasible because of the heterogeneity of the Internetworking devices.

RSVP-TE [17] is traffic engineering version for RSVP and has been used by multiprotocol label switching (MPLS) for traffic engineering in IP networks. RSVP is intended to support many to-many multicast QoS reservations [1]. The authors have summarized the key differences between RSVP and NSIS in a tabular form. Table 1 compares key features of RSVP and NSIS. From the table we could see that RSVP is single layer, receiver initiated, no mobility support and supports multicasting. On the other hand, using a modular approach, NSIS has been split into two layers. Modular approach generally makes it easier to implement a scheme and increase scalability. It could accommodate services requests from senders and receivers, has built-in mobility support and no support for multicasting at the moment. One reason for no multicast support by NSIS would be the complex operation involved in multicast management and especially accommodating multicast during mobility support. RSVP uses general transport protocols like IP/UDP while NSIS uses reliable protocols such as TCP, STCP with support for UDP and DCCP.

TABLE I: SUMMARY OF THE BASIC FEATURES OF RSVP AND NSIS (QOS) SIGNALING

\begin{tabular}{|c|c|c|}
\hline & RSVP & NSIS \\
\hline $\begin{array}{l}\text { Protocol } \\
\text { Structure }\end{array}$ & Single Layer & Two Layers \\
\hline Transport & IP or UDP & $\begin{array}{l}\text { Reliable(TCP,STCP)/Dat } \\
\text { agram(UDP,DCCP) }\end{array}$ \\
\hline $\begin{array}{l}\text { Reservation } \\
\text { Initiator }\end{array}$ & Receiver & Sender or Receiver \\
\hline States & $\begin{array}{l}\text { Soft }+ \text { expl. } \\
\text { Release }\end{array}$ & Soft + expl. Release \\
\hline QoS Models & $\begin{array}{l}\text { IntServ/DiffSer } \\
\mathrm{v}\end{array}$ & IntServ/DiffServ/Other \\
\hline $\begin{array}{l}\text { Scope of } \\
\text { Signaling }\end{array}$ & End-to-End & $\begin{array}{l}\text { End-to-End/Host-to- } \\
\text { Host/Edge-to-Edge }\end{array}$ \\
\hline Multicast & Yes & No \\
\hline Mobility & No & Yes \\
\hline Bi-Directional & No & Yes \\
\hline Aggregation & Yes & Yes \\
\hline $\begin{array}{l}\text { Summary } \\
\text { Refresh }\end{array}$ & Yes & Yes \\
\hline $\begin{array}{l}\text { Priority/preemp } \\
\text { tion }\end{array}$ & Yes & Yes \\
\hline
\end{tabular}

Looking at the literature survey, the need of a more generic and flexible QoS based protocol is quite evident. IETF's NSIS was the effort to provide such architecture and a lot of research is directed to the implementation and optimal use of the NSIS protocol in relation to end-to-end QoS in future networks. The following section looks into the NSIS architecture and some extensions that have been proposed in literature to make NSIS the NGN standard QoS protocol.

\section{NSIS ARCHITECTURE}

We will discuss the NSIS architecture first with a brief introduction of its two layers. Then the section below will explain the NSLP. In the later discussion we will also see works from other researchers using NSIS as QoS for different type of networking environment. The NSIS architecture is conceptually depicted in Figure2 [4].

NSIS had to be modular and to meet this requirement, the NSIS protocol suite has been divided into two layers, namely, 'signaling transport' layer, that will carry signaling messages from source to destination and a 'signaling application' layer, which will handle message formats and sequences related to a specific signaling application. NSIS Transport Layer Protocol(NTLP) refers to the component that will be used in the transport layer and 'NSIS Signaling Layer Protocol' (NSLP) refers generically to any protocol within the signaling application layer; in the end, there will be several NSLPs, largely independent of each other [2].

The lower layer is usually taking care of NSIS signaling. The upper layer is reliant on some signaling application, e.g. resource reservation or NAT and firewall settings [4]. NSIS signaling facilitates the Internet in many ways. This allows the signaling to be originated and aborted in various locations in the network such as end hosts, domain boundaries, and interior routers. It supports end-to-end signaling where the messages exchange is done between end clients, edge-to-edge signaling, and end-to-edge signaling as well. 


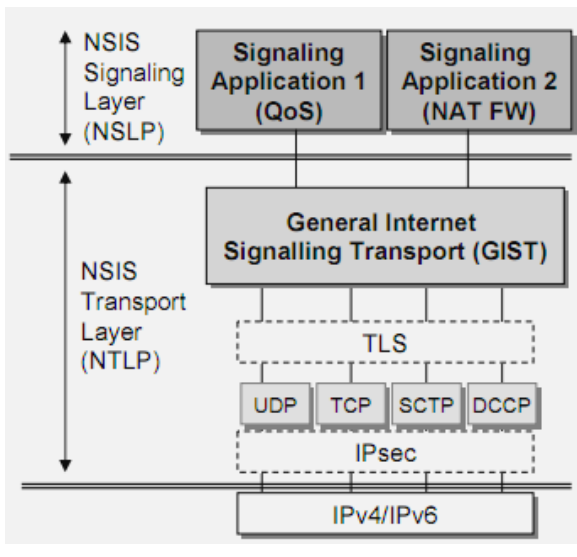

Fig. 2. NSIS Conceptual Architecture

\section{A. The General Internet Signaling Transport Protocol} (GIST)

The authors of [18] have presented a protocol stack for the routing and transport of signaling messages based on per-flow service. They presented GIST, which provides single platform for different kinds of signaling applications. It administers its own internal state and the configuration of the underlying transport and security protocols. This in turn will enable the transfer of messages in both directions along the data flow path. GIST has two modes of operation: the datagram mode, which uses UDP for instance as an unreliable/unsecured datagram transport protocol; and the connection mode, which uses a stream or message based transport protocol, e.g. TCP [1].

Jointly GIST and the lower layer transport and security protocols give a resolution for the base protocol component of the "Next Steps in Signaling" framework. GIST will not set up or modify paths itself; therefore it is paired to protocols like RSVP-TE [17] rather than a substitute [18]. GIST facilitates upper-layer signaling protocols with a few services, which include signaling peer discovery, multiplexing, transport, security, routing changes, fragmentation, state maintenance. Further details on these services could be found in [19].

\section{B. QOS Signaling Application Protocol in NSIS (QoS} $N S L P)$

The NSIS Signaling Layer Protocol (NSLP) for signaling Quality of Service (QoS) reservations in the Internet has been presented in [20]. Working with GIST, it works same as RSVP and also enhances it functionalities. Supporting different reservation models, the QoS NSLP works independent of the underlying QoS specification or architecture. The working of this protocol is much simpler at the moment, because it doesn't support multicast flows. It provides the forwarding resources for a flow by establishing and maintaining the state at different clients within the data path.

QoS NSLP uses soft-state peer-to-peer refresh messages as the main state management method. The QoS NSLP widens the set of reservation mechanisms to meet the requirements of [3], providing support of sender- or receiver-initiated reservations, as well as a type of bidirectional Reservation. It also renders resource reservations between random nodes, e.g., edge-to-edge, end-to-access (for instance, an end user trying to access network services in mobile and wireless environment). At the moment it doesn't support IP multicast. Figure 3 shows the components of QoS NSLP signaling session. The flowsender and flow-receiver would in most cases be part of the QNI and QNR nodes, but they could be separate nodes as well [20]. The terms used in the model are:

QNE: an NSIS Entity (NE), which is QoS NSLP aware.

QNI: the first node in the chain of QNEs that initiates request for a session reservation.

QNR: the last node in the chain of QNEs, which gets a request for session reservation.

QoS NSLP messages are forwarded peer-to-peer, which means that a QNE considers its neighbors to be the source of every message.

A header value depicts the message type and uses some flag bits. QoS NSLP messages contain three types of objects namely:

1) Control Information Objects that carry general information for the QoS NSLP processing, e.g. sequence numbers or response required for the request.

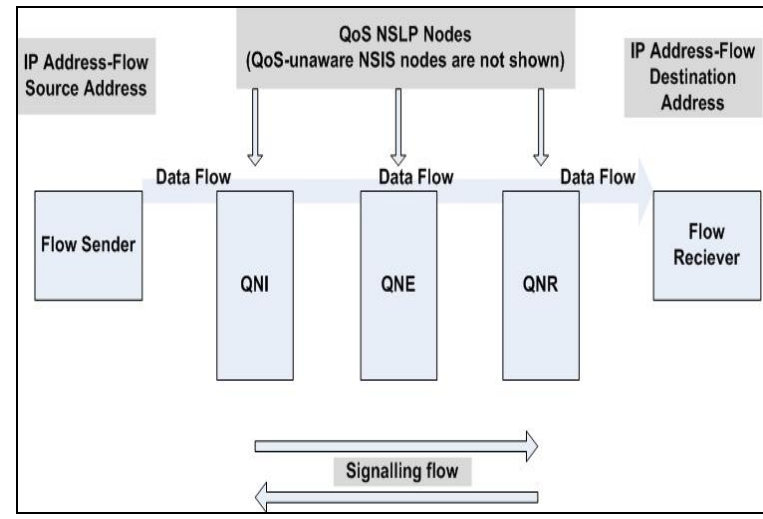

Fig. 3. Components of the QoS NSLP

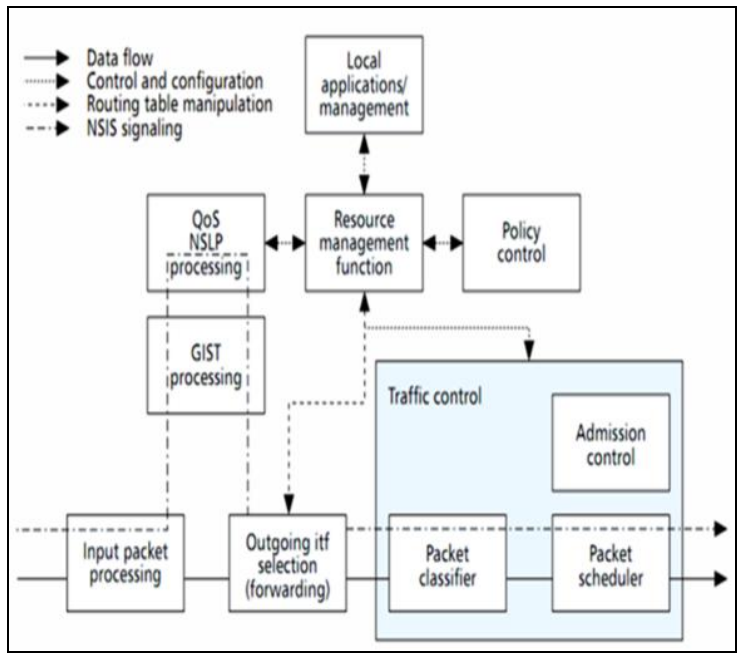

Fig. 4. QoS NSLP model of operation

2) QoS specifications (QSPECs) Objects describing the actual resources that are needed and depend on the QoS model being used.

3) Policy Objects that would contain data used for the authorization of the resource reservation.

Fig. 4 shows the QoS NSLP model of operation. The Figure shows the data flows, control and configuration component and traffic control component to be the core of this model. GIST and QoS NSLP could be seen in the model 
as the core NSIS functional entities [19].

The functionalities of QoS NSLP have been summarized in [19]. Here is the summary of few of the important things that the authors mentioned during their studies:

- Resource requests forwarding for unicast flows

- Decouple the Resource requests (QSPEC) that originate from the signaling protocol (i.e. QoS NSLP)

- Bidirectional and Sender- and receiver-initiated reservations as well

- Message re-ordering and duplication protection

- Rerouting support, for example, nodes mobility

According to M. Arumaithurai et.al, issues like route changes and GIST update delay need to be researched further. QNE proved to be a bottleneck as well. Enabling QoS-NSLP for a better response to route changes is still a gap to be filled with better model to handle such scenarios [21].

\section{Possible NSIS EXTENSIONS}

J. Manner et.al. [19] Have drafted suggestions on how the industry and community can make use of the new protocols and how to utilize the extensibility of NSIS framework and existing protocols to address future signaling needs. Below is a brief summary of key achievements of their work [19].

\section{A. NAT/Firewall Traversal NSLP}

The NAT/firewall traversal NSLP [22] allows end-hosts to talk to NAT and firewall devices in the data path. Signaling messages may traverse many NAT/firewall-NSLP aware (NSIS Forwarder) middleboxes to reach the end nodes. In cases where the NAT/firewall NSLP has not a fully supported data path, proxy mechanism would be available. NAT/firewall NSLP is soft-state protocol as described earlier.

\section{B. GIST Extensions}

One required future extension to GIST is catering the multi-cast flows and extending support to scenarios where end-to-end data flow signaling is not required. Message routing method (MRM) handles routing of GIST messages through the network. Two routing methods that are being used are, Path Coupled MRM and Loose End MRM, so there is room to work out more MRMs [22].

The authors have listed a few opportunities that could be possible for the future extensions in NSIS framework. We are listing some for brevity sake, please see [22] for more details on these possible extensions:

- Using Different Security Services

- Exploring alternate Message Routing Methods

- Trying diverse Transport Protocols or Security facilities

- Query Mode Packet Interception Schemes

- Using other available NAT Traversal Mechanisms

- More Error Identification flags

- Defining New Objects To Be Carried in GIST

- Additional Message Types

- QoS NSLP
- QoS Specifications

- New NSLP Protocols

- NAT/Firewall NSLP

- Reviewing Security

\section{CONCLUSION}

Working on NSIS since 2001 the IETF has been successful in designing and developing the NSIS protocol suite. The development of NSIS protocol has enabled the research community to move in the right direction in order to attain the end-to-end QoS provision in future generation networks or NGN. The generic approach and extensibility of the NSIS protocol suite is of great interest to research community as a resort for provision of QoS in number of internet communication scenarios like mobile, wireless, adhoc networks and fixed networks. The researchers of NGN can definitely get a great benefit with the flexible nature of the protocol and help design a more reliable and better QoS implementation model in the near future. The possible future extensions summary presented in this paper can lead us to creation of better solutions for NGN QoS.

\section{REFERENCES}

[1] X. Fu, H. Schulzrinne, A. Bader, D. Hogrefe, C .Kappler, H. Tshofenig, and S. Bosch, "NSIS: A New Extensible IP Signaling Protocol Suite," IEEE Communications Magazine, pp. 133-141, Oct 2005.

[2] R. Hancock, G. Karagiannis, J. Loughney, and S. Van den Bosch. (June 2005). Next Steps in Signaling (NSIS): Framework. RFC 4080. [Online]. Available: http://datatracker.ietf.org/doc/rfc4080/

[3] M. Brunner. (April 2004). Requirements for Signaling Protocols. RFC 3726. [Online]. Avaialble: http://datatracker.ietf.org/doc/rfc3726/

[4] R. Bless and M. Rohricht, "End-to-End Quality-of-Service Support in Next Generation Networks with NSIS," IEEE International Conference on Communications (ICC), May 2010.

[5] M. Waskasi, M. Sadeghi, M. Mirzabaghi, Y. Harandi, A. Tabrizipoor and M. Pirhadi, "NGN Test Strategy: Evaluating Next Generation Networks in a Realistic Environment," in Proc. Innovations in NGN: Future Network and Services, 2008. K-INGN 2008. First ITU-T Kaleidoscope Academic Conference, 12-13 May 2008, pp. 323-330.

[6] M. Shakir, "Challenging Issues in NGN Implementation and Regulation," in Proc. 6th International Conference on Wireless Communications Networking and Mobile Computing (WiCOM), 2325 Sept. 2010, pp. 1-4.

[7] O. Friedrich, A. Al-Hezmi, and T. Magedanz, "Evolution of Next Genration Networks Towards an integrated platform for IMS-based IPTV services," International Symposium on Applications and the Internet Workshops, 2007.

[8] A. Darvisham, H. Yeganeh, K. Bamasian, and P. Eghtedari, "A practical NGN Model By Evaluation of Various NGN Soultions and its Conformance with IMS-TISPAN," in Proc. the 2nd IEEE International Conference on Information Management and Engineering (ICIME), 16-18 April 2010, pp. 59-63.

[9] El-B. Fgee, J. Kenney, W. Phillips, W. Robertson and S. Sivakumar, "Comparison of QoS performance between IPv6 QoS management model and IntServ and DiffServ QoS models," in Proc. the IEEE 3rd Annual Communication Networks and Services Research Conference, 16-18 May 2005, pp. 287 - 292.

[10] J. Harju and Perttu, "Co-operation and Comparison of DiffServ and IntServ: Performance Measurements," in Proceedings of 25th Annual IEEE Conference on Local Computer Networks, 2000. pp. 177 - 186.

[11] E.-H. Cho, K.-S. Shin, and S.-J. Yoo, "SIP-based Qos support architecture and session management in a combined IntServ and DiffServ networks," Computer Communications, vol. 29, pp. 29963000, Elsevier, 2006.

[12] Z. Mammeri, "Framework for parameter mapping to provide end-toend QoS guarantees in IntServ/DiffServ architectures," $A C M$ Computer Communications, vol. 28, issue 9, 2 June 2005, pp. 10741092 .

[13] S. Park, "DiffServ Quality of Service Support for Multimedia Applications in Broadband Access Networks," presented at IEEE 
International Conference Hybrid Information Technology 2006, 9-11 Nov. 2006.

[14] M. Á. Callejo-Rodríguez and J. Enríquez-Gabeiras, "Bridging the Standardization Gap to Provide QoS in Current NGN Architectures,' IEEE Journals, Communications Magazine, vol. 46, issue 10, pp. 132 - 137, October 2008

[15] J. Manner and X. Fu (May 2005). Analysis of Existing Quality-of Service Signaling Protocols. RFC 4094. [Online]. Avaialble: http://www.rfceditor. org/rfc/rfc4094.tx

[16] S. Wei, Z. Xue and W. Xiaochun, "Design and Implementation of a REVP-Capable router for multiple QoS support," in proceedings of International Conference on Computer Networks and Mobile Computing, 2001, pp. 254-259

[17] D. Awduche, L. Berger, D. Gan, T. Li, V. Srinivasan, and G. Swallow (Dec. 2001). RSVP-TE: Extensions to RSVP for LSP tunnels. RFC 3209. [Online]. Available: http://www.rfceditor.org/rfc/rfc3209.txt
[18] H. Schulzrinne and R. Hancock (December 5, 2009). GIST: Genera Internet Signaling Transport. RFC 5971. [Online]. available: http://tools.ietf.org/id/draft-ietf-nsis-ntlp-20.txt

[19] J. Manner, R. Bless, J. Loughney and E. Davies (October 2010). Using and Extending the NSIS Protocol Family. RFC 5978. [Online]. Available: http://datatracker.ietf.org/doc/rfc5978/

[20] J. Manner, G. Karagiannis and A. McDonald. (October 2010). NSIS Signaling Layer Protocol (NSLP) for Quality-of-Service Signaling. RFC $5974 . \quad$ [Online]. Available: https://datatracker.ietf.org/doc/rfc5974/

[21] M. Arumaithurai, X. Fu, B. Schloer and H. Tschofenig, "Performance Study of the NSIS QoS-NSLP Protocol," in Proc. IEEE Global Telecommunications Conference, 2008, pp. $1-6$.

[22] M. Stiemerling, H. Tschofenig, C. Aoun and E. Davies (October 2010). NAT/Firewall NSIS Signaling Layer Protocol (NSLP). RF 5973. [Online]. Available: http://datatracker.ietf.org/doc/rfc5973/ 